\title{
Dynamic Characterization of Microcantilevers with a Shock Wave Excitation Method under High Temperature
}

\author{
Dongsheng She ${ }^{1, *}$ - Yiliu Yang 2 - Zefei Wei ${ }^{1}$ - Zhen Yu ${ }^{1}$ \\ 1Bohai University, College of Engineering, China \\ 2Bohai University, Research and Teaching Institute of College Computer Science, China
}

\begin{abstract}
Aiming at the dynamic characteristics of silicon microcantilevers under a high-temperature environment, a shock wave excitation method was proposed, and a dynamic testing system with high-temperature loading unit for MEMS microstructures was established. In the system, the shock wave generated by electrical discharging was used to excite the testing microcantilever. The vibration response signals were acquired by laser Doppler vibrometer system. A T-shaped microcantilever and a microcantilever with uniform rectangular crossing section were fabricated and tested under the high-temperature environment ranging from $299 \mathrm{~K}$ to $773 \mathrm{~K}$. Their temperature coefficients of natural frequency were obtained. The results show that for both two microcantilevers, the temperature coefficients of the natural frequency is very close to each other, which is only decided by the temperature coefficient of the elastic modulus and the linear thermal expansion coefficients.

Keywords: dynamic characteristics testing, microcantilever, shock wave, temperature coefficient of natural frequency, high temperature

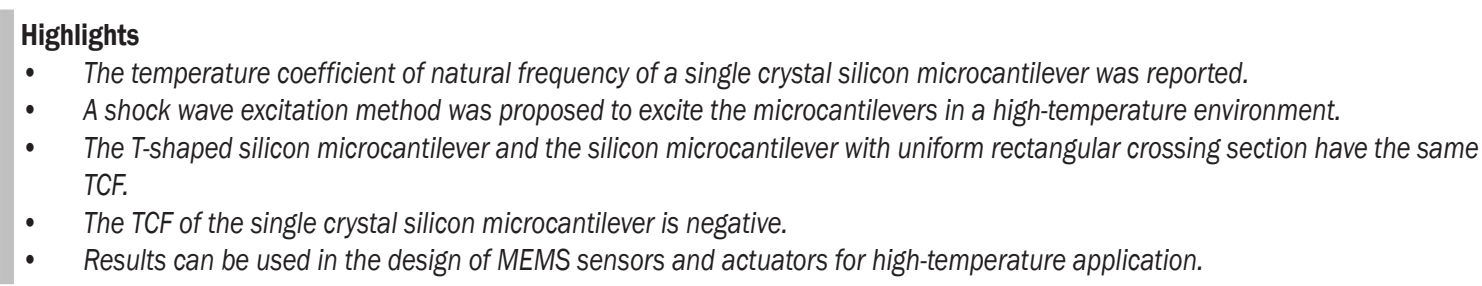

\section{O INTRODUCTION}

Microelectromechanical system (MEMS) sensors and actuators have shown great potential in many areas including automotive, aerospace, missile weapon and nuclear power, etc. [1] and [2]. Thus, there is a need for MEMS devices to work in high-temperature environments. Because the performance of MEMS devices is strongly influenced by the dynamic characteristics of its internal microstructures, studying the dynamic characteristics of microstructures under high-temperature environments is urgently needed.

The temperature coefficient of natural frequency (TCF) is an important parameter for MEMS design. Based on the TCF, the variation tendency of resonant frequencies for microstructures can be predicted when the temperature changes. Many researchers have studied the TCFs of basic MEMS microstructures. Sandberg, et al. tested the TCF of the first five modes of $\mathrm{SiO}_{2}$ microcantilevers under the temperature ranging from $303 \mathrm{~K}$ to $353 \mathrm{~K}$. They found that the TCF of the uncoated $\mathrm{SiO}_{2}$ microcantilever is a constant of ca. $9.79 \mathrm{E}-5 \mathrm{~K}^{-1}$ [3]. However, the TCF of the goldcoated $\mathrm{SiO}_{2}$ microcantilever is highly irregular for different modes. Boyd, et al. studied the TCFs of single crystal silicon microcantilevers and $3 \mathrm{C}$ silicon carbide microcantilever at temperatures ranging from
$200 \mathrm{~K}$ to $273 \mathrm{~K}$, respectively. They found that the TCF of single crystal silicon microcantilevers is about $-2.47 \mathrm{E}-5 \mathrm{~K}^{-1}$, and the TCF of $3 \mathrm{C}$ silicon carbide microcantilevers is about $-1.8 \mathrm{E}-5 \mathrm{~K}^{-1}$ [4]. Chuang tested the T-shaped silicon nitride microcantilevers under a cryogenic temperature environment. They found that there was a slight decrease in the resonance frequency when the temperature was elevated from $30 \mathrm{~K}$ to $298 \mathrm{~K}$. The TCF of the T-shaped silicon nitride microcantilevers are $-5.65 \mathrm{E}-5 \mathrm{~K}^{-1}$ [5]. It can be seen that there are great differences in TCF for different materials.

To obtain the TCFs of microstructures experimentally, a dynamic testing system with a temperature loading unit is needed. For the last three decades, much effort has been made on developing methods and techniques for the dynamic testing of microstructures. The dynamic testing techniques for microstructures mainly include the vibration measurement techniques and the excitation methods. Because the microstructures have the features of small size, light weight, and high natural frequency, conventional measurement methods cannot be applied without modification. So far, the vibration measurement techniques for microstructures can be divided into two types: built-in self-test (BIST) methods and non-contact optical methods. The BIST 
methods need to integrate the embedded sensing elements, such as piezoresistive elements [6] and capacitive elements [7], into the microstructures. The non-contact optical methods, such as laser Doppler vibrometer (LDV) [8], stroboscopy and interferometry technique [9] and electronic speckle pattern interferometry (ESPI) [10], have several advantages over the BIST methods. Since there are no additional elements needed, the dynamic characteristics of the original microstructures will not be modified. In particular, laser Doppler vibrometer is widely used in out-of-surface vibration measurement due to its high accuracy. Regarding excitation methods, there are three categories: excitation method with embedded elements [11] to [13], non-contact excitation method [14] to [16] and base excitation method with a bulk piezoelectric ceramic transducer (PZT) [17] and [18]. Compared to other excitation methods, base excitation with bulk PZT is non-destructive, simple, and suitable for microstructures with high resonant frequency. However, this method cannot be used in hightemperature environments, because the piezoelectric ceramic is non-effective when the temperature is elevated approaching its Curie point.

In this paper, the TCF of single crystal silicon microcantilevers was studied under high temperatures ranging from $299 \mathrm{~K}$ to $773 \mathrm{~K}$. A dynamic testing system with a high-temperature loading unit was established. In the system, an impact base excitation device with shock wave was developed to excite the microstructures. A LDV was used to obtain the vibration response signals of microstructures. A silicon microcantilever with uniform rectangular crossing section and a T-shaped silicon microcantilever were fabricated. The TCFs of two microcantilevers were tested, and the results were discussed.

\section{METHOD}

An impact base excitation method with shock wave was proposed to excite the microcantilevers. In this method, the testing microcantilever is attached on one side of a base structure that is installed on a rigid platform. A plate electrode is attached on the other side of the base structure. A needle electrode is fixed on a feeder. The distance between two electrodes can be changed by adjusting the feeder. Two electrodes are wired to the two poles of a capacitor. A direct current (DC) high voltage supply is used to charge the capacitor. The shock wave can be generated when the distance between two electrodes meets the requirement of electrical discharging. The base structure will be impacted by the action of the shock wave. The microcantilever can be excited by the movement of the base structure.

The laser Doppler vibrometer technique is used to obtain the vibration response signals of microcantilevers. The principle of this method is as follows: when a laser is projected to the surface of a moving object, the Doppler effect will occur. The frequency of the reflected light will be changed. The frequency shift is related to the moving speed of the object, and can be written by:

$$
f_{D}=\frac{2 v}{c-v}
$$

where $f_{D}$ is the frequency shift of the reflected light, $c$ is the speed of the light and $v$ is the moving speed of the object. The moving speed of the object can be obtained if the frequency shift has been measured. Furthermore, the vibration parameters of the object can also be obtained based on its moving speed.

\section{TESTING SYSTEM SETUPS}

A dynamic testing system was established to measure the dynamic characteristics of microstructures under the high-temperature environment ranging from $299 \mathrm{~K}$ to $773 \mathrm{~K}$. In the system, an impact base excitation device with shock wave was developed to excite the microcantilevers. A laser Doppler vibrometer was employed to detect the vibration response signals of the microcantilevers.

\subsection{Excitation Device}

An excitation device was developed basing on the principle of the impact base excitation method with a shock wave, as is shown in Fig. 1.

Five high voltage capacitors were connected in parallel with each other to provide a high impact voltage power. The capacitance of each capacitor is $0.22 \mu \mathrm{f}$. The discharging electrodes were fabricated with $\mathrm{Wu}-\mathrm{Cu}$ alloy material due to its high-temperature resistance and ablative resistance. A crossing leaf spring was used as the base structure. A straight micrometer was chosen as the feeder to adjust the distance between the two electrodes. A ceramic disc was attached between the crossing leaf spring and the plate electrode for the electrical insulation. For the same purpose, a ceramic tube was installed between the needle electrode and the straight micrometer. 


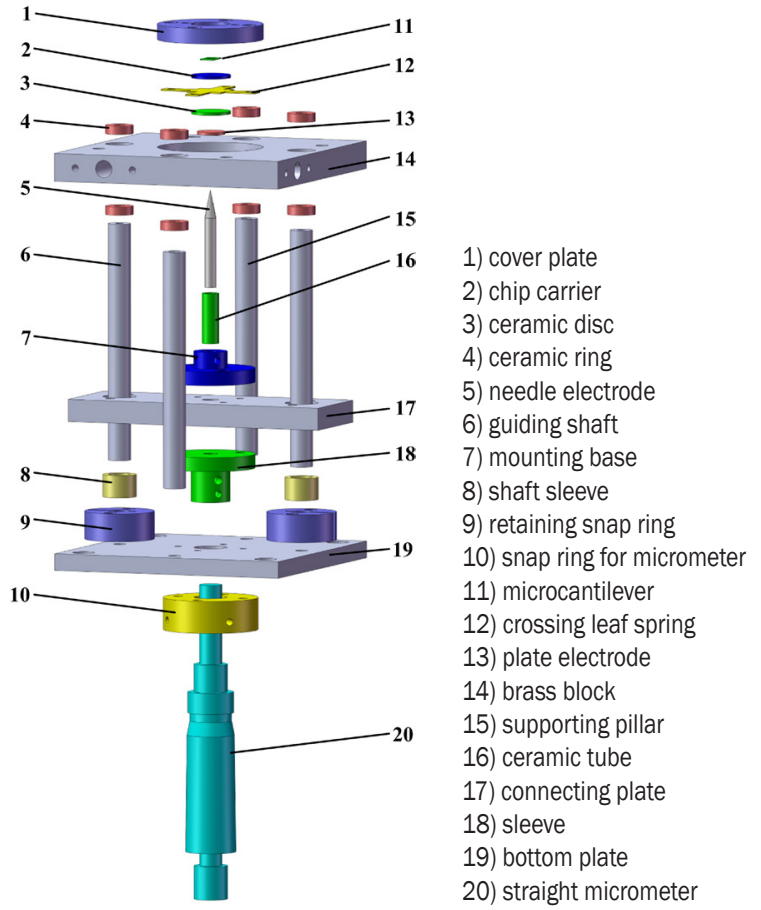

Fig. 1. Exploded views of excitation device

\subsection{Data Acquisition and Analysis System}

A Polytec OFV-534 laser Doppler vibrometer system was employed to measure the dynamic characteristics of microstructures. The system mainly consists of a compact vibrometer sensor head, a CCD camera, a He-Ne laser, an illumination unit, a VDD-E-600 digital front end, a PCI-6110E date acquisition card, and VIBSOFT-VDD vibration measurement software.

The principle of the OFV-534 laser Doppler vibrometer system is as follows: the He-Ne laser emits a $632 \mathrm{~nm}$ laser into the compact vibrometer sensor head, in which the laser is split into two laser beams. One is used as the reference laser; the other is used as the incident laser which is irradiated on the testing microcantilever. As soon as the microcantilever vibrates, due to the Doppler effect, the reflected laser contains the frequency signal of the microcantilever. The reflected laser enters the compact vibrometer sensor head in which the reflected laser interferes with the reference laser. Then the interference signal is fed into the VDD-E-600 digital front end, and converted to a standard electrical signal. The obtained standard electrical signal is read through the PCI-6110E data acquisition card, using the VIBSOFT-VDD vibration measurement software. Thus, the vibration response signal of the testing microcantilever is obtained.
As shown in Fig. 2, the compact vibrometer sensor head was mounted on a translation and rotation stage, which contains three translational degrees of freedom and two rotational degrees of freedom. With the aid of the CCD camera and the illumination unit, the laser spot can be positioned on the testing microcantilever easily and precisely by adjusting the translation and rotation stage.

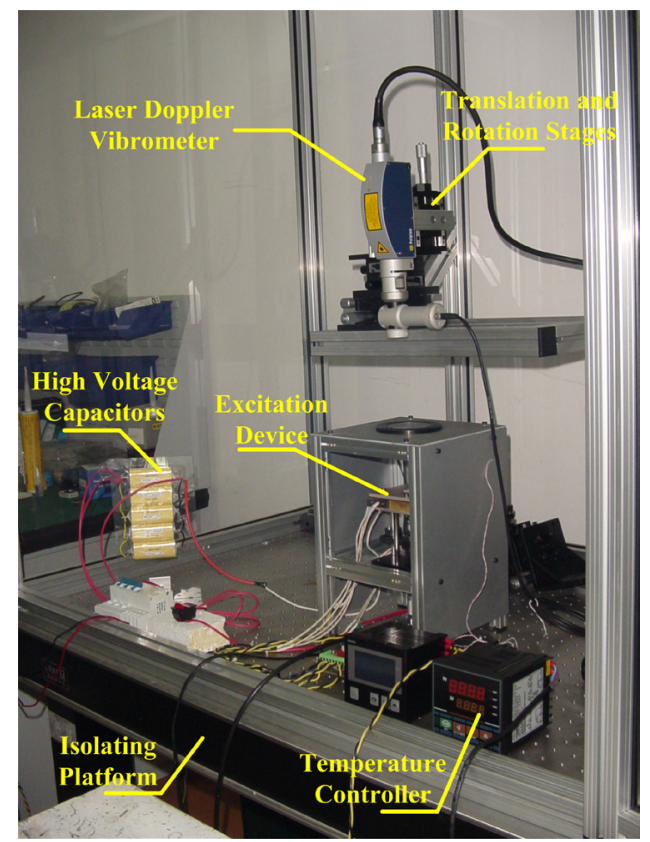

Fig. 2. Dynamic testing system for microstructures with hightemperature loading unit

\subsection{High-Temperature Environment}

Four resistive heaters were installed into the brass block, which can change the temperature of the microcantilever from room temperature $299 \mathrm{~K}$ up to $773 \mathrm{~K}$. The heat transferred from the heaters to the testing microcantilever through the brass block and the crossing leaf spring. The excitation device was installed in a protective chamber to decrease the heat loss. Through the quartz glass window on the top of the chamber, the dynamic response of the testing microcantilever can be obtained by the laser Doppler vibrometer.

\section{MICROCANTILEVERS}

Two single crystal silicon microcantilevers were fabricated and named Microcantilever \#1 and Microcantileva \#2. Microcantileva \#1 is a T-shaped silicon microcantilever, and Microcantileva \#2 is a 
microcantilever with uniform rectangular crossing section. Their scanning electron microscope (SEM) photographs were shown in Fig. 3.
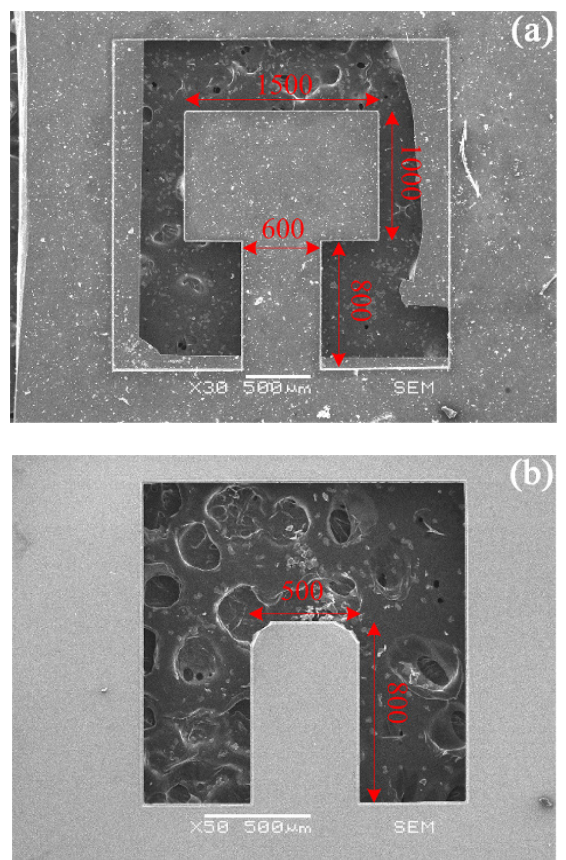

Fig. 3. SEM photos of microcantilevers. a) \#1, b) \#2

The dimensions of Microcantileva \#1 are as follows: $l_{\text {beam }}$ is the length of the microcantilever beam, which is equal to $800 \mu \mathrm{m}$. w $w_{\text {beam }}$ is the width of the microcantilever beam, which is equal to $600 \mu \mathrm{m}$. $l_{\text {mass }}$ is the length of the proof mass, which is equal to $1000 \mu \mathrm{m} . w_{\text {mass }}$ is the width of the proof mass, which is equal to $1500 \mu \mathrm{m} . h_{1}$ is the thickness of the microcantilever, which is equal to $10 \mu \mathrm{m}$.

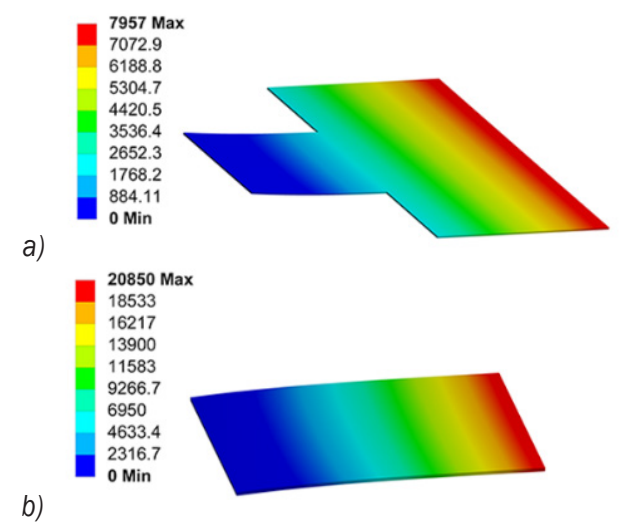

Fig. 4. Finite element analysis results of natural frequencies at the room temperature. a) \#1, b) \#2

The dimensions of Microcantileva \#2 are as follows: $l$ is the length of the microcantilever, which is equal to $800 \mu \mathrm{m} . w$ is the width of the microcantilever, which is equal to $500 \mu \mathrm{m} . h_{2}$ is the thickness of the microcantilever, which is equal to $10 \mu \mathrm{m}$.

The first-order natural frequencies of both microcantilevers in $293 \mathrm{~K}$ were simulated with ANSYS software. The reference values of material properties of silicon are as follows. The elastic modulus $E$ is equal to $167 \mathrm{GPa}$. The density $\rho$ is equal to $2330 \mathrm{~kg} / \mathrm{m}^{2}$. Poisson's ratio is equal to 0.278 [19]. The results are shown in Fig. 4. It is $2.7995 \mathrm{kHz}$ for Microcantileva \#1, and $21.988 \mathrm{kHz}$ for Microcantileva $\# 2$.

\section{EXPERIMENTS AND DISCUSSION}

\subsection{Natural Frequency at Room Temperature}

Two microcantilevers were tested with the developed dynamic testing system at room temperature. The obtained vibration response signals are shown in Figs. 5 and 6, respectively. Applying FFT to these signals, the frequency domain signals were obtained, as shown in Figs. 7 and 8, respectively.

In Fig. 7, there are two distinct peaks at $2.692 \mathrm{kHz}$ and $45.632 \mathrm{kHz}$. In Fig. 8, there are two distinct peaks at $20.712 \mathrm{kHz}$ and $45.649 \mathrm{kHz}$. According to the finite element analysis (FEA) results of two microcantilevers in Section 3, it can be inferred that the first-order resonant frequency of Microcantileva \#1 is $2.692 \mathrm{kHz}$, and the first-order resonant frequency of Microcantileva \#2 is $20.712 \mathrm{kHz}$. The common frequency peak, $45.6 \mathrm{kHz}$, may be the resonant frequency of the base structure or the resonant frequency of the substrate of the silicon chip. The firstorder resonant frequencies of the microcantilevers can be considered to be approximately equal to their firstorder natural frequencies because the air damping is negligible compared with the structural damping.

There are some differences between the obtained natural frequencies and the FEA results. The following reasons may cause the differences. Firstly, the material properties, such as elastic modulus, density, and Poisson's ratio, will be changed by the fabrication process of silicon microcantilevers. Thus, the actual values of silicon material parameters are different from the selected values in FEA. Secondly, the actual boundary conditions of microcantilevers are different from the ideal conditions. Thirdly, the dimensions of microcantilevers are different from the theoretical values, such as the length, the width, the thickness and the shape of the crossing section. Finally, the measurement error is another affecting factor. 


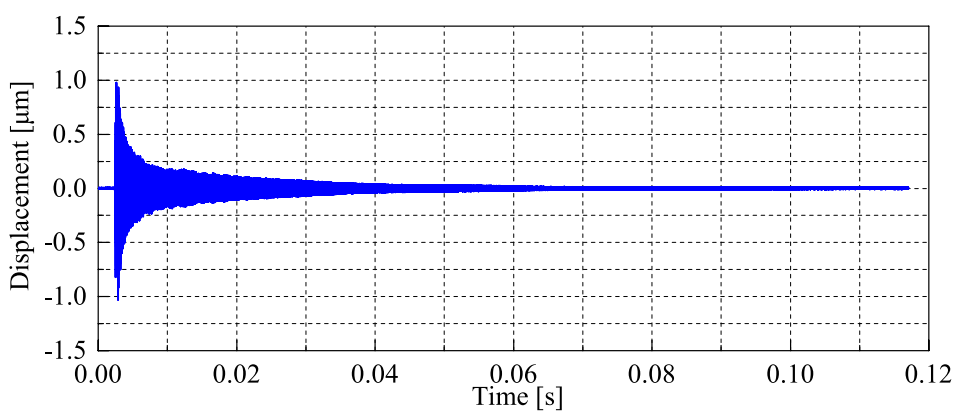

Fig. 5. Vibration response signal of Microcantileva \#1

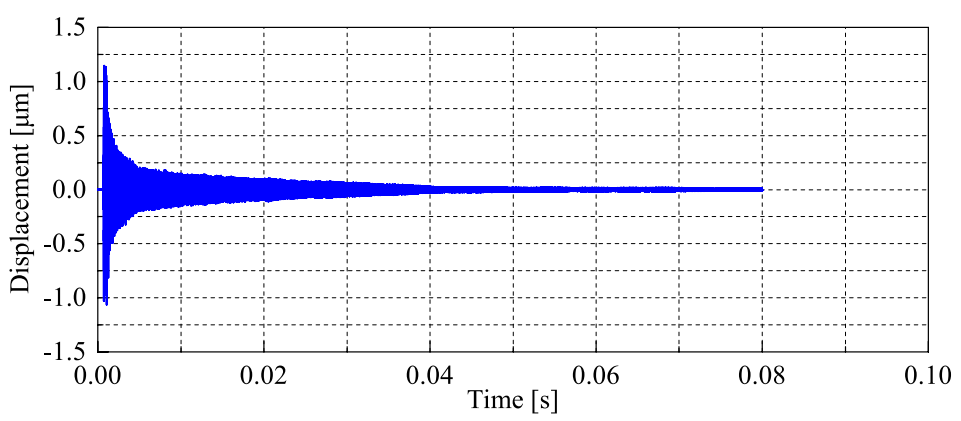

Fig. 6. Vibration response signal of Microcantileva \#2

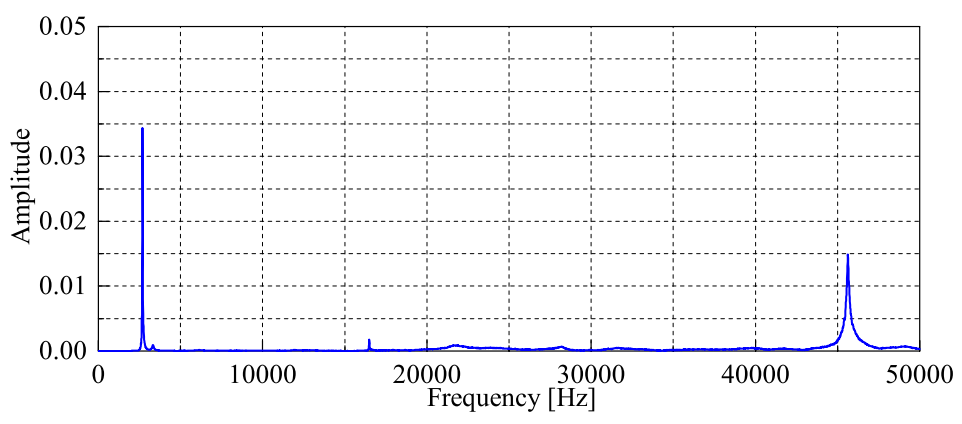

Fig. 7. Frequency spectrogram of Microcantileva \#1

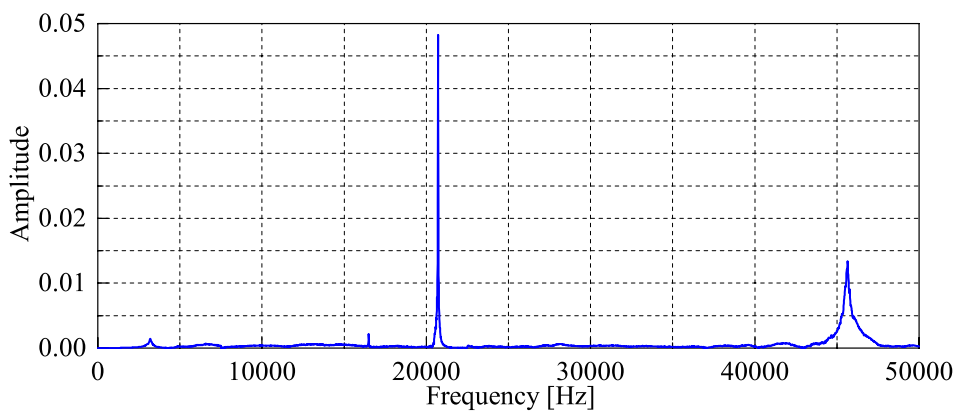

Fig. 8. Frequency spectrogram of Microcantileva \#2

\subsection{Natural Frequency versus Temperature}

The dynamic testing experiment was carried out under a high-temperature environment ranging from room temperature to $773 \mathrm{~K}$, and the results are shown in Figs. 9 and 10.

It can be seen that for both microcantilevers, their first-order natural frequencies slightly decrease 
with the elevated temperature. Furthermore, there is an almost linear dependence between the first-order natural frequency and the temperature. The rate of change is about $-0.069 \mathrm{~Hz} / \mathrm{K}$ for Microcantileva \#1, and $-0.51 \mathrm{~Hz} / \mathrm{K}$ for Microcantileva \#2.

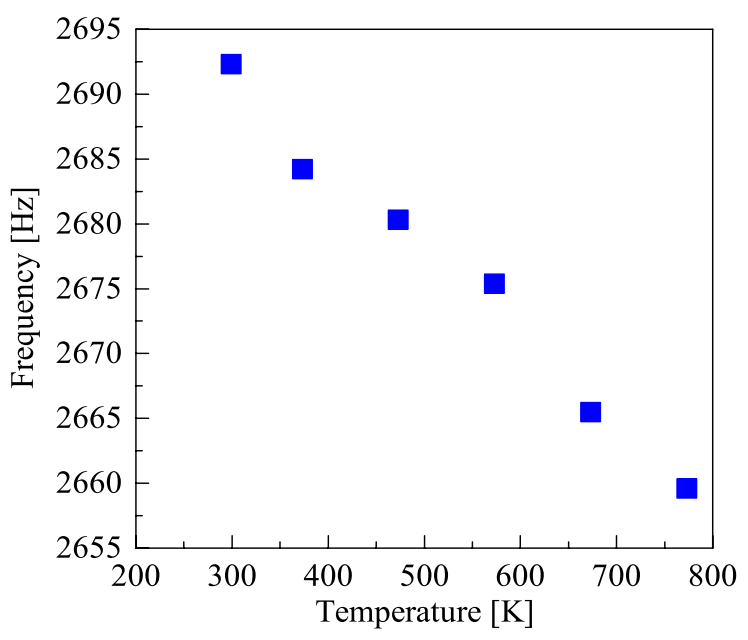

Fig. 9. The first-order natural frequency of Microcantileva \#1 at the different testing temperature

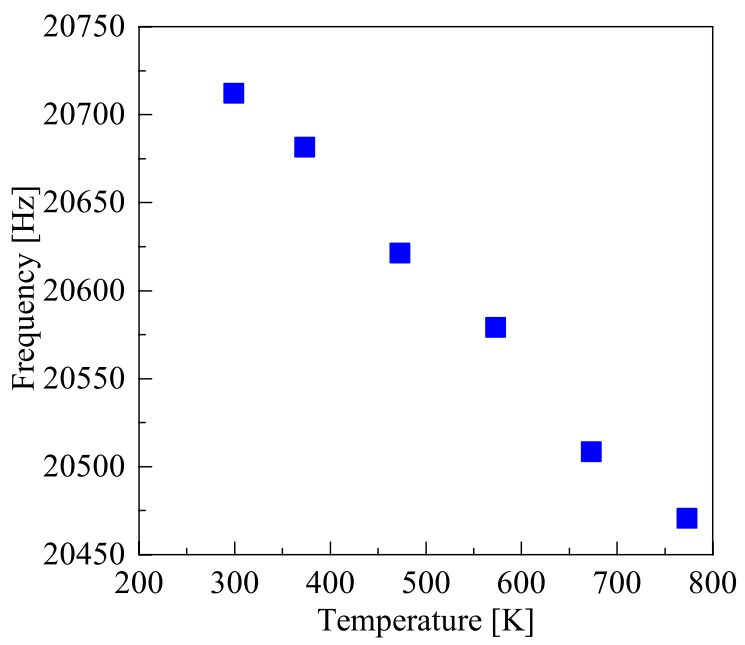

Fig. 10. The first-order natural frequency of Microcantileva \#2 at the different testing temperature

To determine the TCFs of microcantilevers, the normalized natural frequencies of two microcantilevers were calculated and are shown in Fig. 11. For each microcantilever, its TCF is the slope of the corresponding linear fitting line. It can be seen that the two microcantilevers almost have the same TCF. The obtained TCF is $-2.56 \mathrm{E}-5 \mathrm{~K}^{-1}$ for Microcantileva $\# 1$, and $-2.46 \mathrm{E}-5 \mathrm{~K}^{-1}$ for Microcantileva $\# 2$. They are very close to the value reported in reference [11]. To figure out why both two different shaped microcantilevers have the same TCF, the theoretical TCFs for both microcantilevers were analysed as follows.

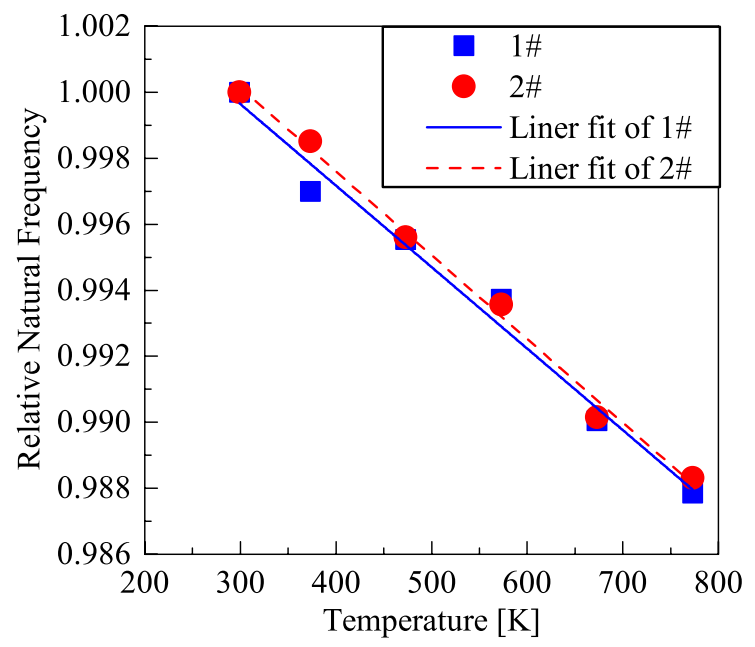

Fig. 11. Normalized natural frequencies of microcantilevers as functions of temperature

Generally, the TCF can be determined by:

$$
T C F=\frac{1}{f\left(T_{0}\right)} \cdot \frac{\partial f(T)}{\partial T},
$$

where $T_{0}$ is the starting temperature, $f\left(T_{0}\right)$ means the reference natural frequency.

For the microcantileva \#1, its first-order natural frequency can be expressed as [20]:

$$
f_{1}=\frac{1}{2 \pi} \sqrt{\frac{3 E I_{1}}{L^{3}\left(m_{\text {mass }}+c m_{\text {beam }}\right)}},
$$

where $f_{1}$ is the first-order natural frequency, $E$ is the elastic modulus, $m_{\text {mass }}$ is the proof mass, $m_{\text {beam }}$ is the mass of microcantilever beam, $c$ is a constant that is equal to 0.2357 [21], $L$ is the effective length of the T-shaped microcantilever, and $I_{1}$ is the area moment of inertia. $L$ and $I$ can be determined by Eqs. (4) and (5), respectively.

$$
\begin{gathered}
L=l_{\text {beam }}+\frac{1}{2} l_{\text {mass }}, \\
I_{1}=\frac{1}{12} w_{\text {beam }} h_{1}^{3} .
\end{gathered}
$$

Inserting Eqs. (4) and (5) into Eq. (3), and then introducing the temperature variable $T$ into Eq. (1), the first-order natural frequency of Microcantileva \#1 can be written as: 


$$
\begin{gathered}
f_{1}(T)=k_{1} \cdot E(T)^{\frac{1}{2}} \cdot w_{\text {beam }}(T)^{\frac{1}{2}} \cdot h_{1}(T)^{\frac{3}{2}} . \\
\cdot\left(l_{\text {beam }}(T)+\frac{1}{2} l_{\text {mass }}(T)\right)^{-\frac{3}{2}} .
\end{gathered}
$$

In Eq. (6), $k_{1}$ is a temperature-independent constant, which can be expressed as:

$$
k_{1}=\frac{1}{4 \pi \sqrt{c m_{\text {beam }}+m_{\text {mass }}}} .
$$

Differentiating Eq.(6) with respect to the temperature and dividing by Eq.(6), then inserting $T_{0}$ to the results, the TCF of the T-shaped microcantilever can be obtained.

$$
\begin{aligned}
T C F= & -\frac{3}{2} \cdot \frac{l_{\text {beam }}(T)}{l_{\text {beam }}(T)+l_{\text {mass }}(T) / 2} \cdot \alpha_{L}- \\
& -\frac{3}{4} \frac{l_{\text {mass }}(T)}{l_{\text {beam }}(T)+l_{\text {mass }}(T) / 2} \cdot \alpha_{L}+ \\
& +\frac{3}{2} \cdot \alpha_{h}+\frac{1}{2} \cdot \alpha_{w}+\frac{1}{2} \cdot \beta,
\end{aligned}
$$

where $\alpha_{L}$ is the linear thermal expansion coefficient in the length direction, $\alpha_{h}$ is the linear thermal expansion coefficient in the height direction, $\alpha_{w}$ is the linear thermal expansion coefficient in the width direction, $\beta$ is the temperature coefficient of the elastic modulus. Thus, the TCF of the T-shaped microcantilever can be simplified as:

$$
T C F=-\frac{3}{2} \alpha_{L}+\frac{3}{2} \alpha_{h}+\frac{1}{2} \alpha_{w}+\frac{1}{2} \beta .
$$

For the microcantileva \#2, its first-order natural frequency can be expressed as [20]:

$$
f_{2}=\frac{3.515}{2 \pi} \sqrt{\frac{E I_{2}}{m l^{3}}}
$$

where $m$ is the mass of the microcantilever, $I_{2}$ is the area moment of inertia. $I_{2}$ can be determined by the Eq. (11).

$$
I_{2}=\frac{1}{12} w h_{2}^{3} .
$$

By introducing the temperature variable $T$ into Eq. (10), the first-order natural frequency of Microcantileva \#2 can be written as:

$$
f_{2}(T)=k_{2} \cdot E(T)^{\frac{1}{2}} \cdot w(T)^{\frac{1}{2}} \cdot h_{2}(T)^{\frac{3}{2}} \cdot l(T)^{-\frac{3}{2}} .
$$

In Eq. (12), $k_{2}$ is a temperature-independent constant, which can be expressed as:

$$
k_{2}=\frac{3.515}{4 \pi \sqrt{3 m}} .
$$

Using a similar method as for Microcantilever 1\#, the TCF of Microcantileva \#2 can be obtained.

$$
T C F=-\frac{3}{2} \alpha_{l}+\frac{3}{2} \alpha_{h}+\frac{1}{2} \alpha_{w}+\frac{1}{2} \beta,
$$

where $\alpha_{l}$ is the linear thermal expansion coefficient in the length direction.

It can be seen that for both the T-shaped microcantilever and microcantilever with a uniform rectangular crossing section, the TCF is only decided by the temperature coefficient of the elastic modulus and the linear thermal expansion coefficients. Both microcantilevers were fabricated using a $220 \mu \mathrm{m}$ thick, [100] oriented, single crystal silicon wafer. The length directions of the microcantilevers were all along the [110] crystal orientation. Thus, the same TCF can be obtained.

According to Eq. (10), the first-order natural frequency of Microcantileva \#2 can also be expressed as:

$$
f_{2}=\frac{3.515}{2 \pi \sqrt{12}} \cdot \frac{h}{l^{2}} \cdot \sqrt{\frac{E}{\rho}},
$$

where $\rho$ is the density of the microcantilever. By introducing the temperature variable $T$ into Eq.(15), the first-order natural frequency of Microcantileva \#2 can also be written as:

$$
f_{2}(T)=k_{3} \cdot E(T)^{\frac{1}{2}} \cdot h(T) \cdot l(T)^{-2} \cdot \rho(T)^{-\frac{1}{2}} .
$$

In Eq. (16), $k_{3}$ is a temperature-independent constant, which is approximately equal to 0.162 . Differentiating Eq. (16) with respect to the temperature and dividing by Eq. (16), then inserting $T_{0}$ to the results, the TCF of Microcantilever 2\# can be obtained.

$$
T C F=-2 \alpha_{l}+\alpha_{h}+\frac{1}{2} \beta-\frac{1}{2} \delta,
$$

where $\delta$ is the temperature coefficient of density. As for the single crystal silicon material, its temperature coefficient of elastic modulus is about $-5.2 \mathrm{E}-5 \mathrm{~K}^{-1}$ [22] and [23], and its temperature coefficient of density is about $-1.3 \mathrm{E}-5 \mathrm{~K}^{-1}$ [24]. The linear thermal expansion coefficient in the [110] crystal orientation, $\alpha_{l}$, is almost equal to the linear thermal expansion coefficient $\alpha_{h}$ in the [100] crystal orientation [25]. Moreover, they increase with the elevated temperature. The linear thermal expansion coefficient in the length direction or in the height direction is $2.57 \mathrm{E}-6 \mathrm{~K}^{-1}$ in 
$293 \mathrm{~K}$, and $4.14 \mathrm{E}-6 \mathrm{~K}^{-1}$ in $800 \mathrm{~K}$. Thus, according to the Eqs. (9), (14) and (17), the theoretical TCF of both two microcantilevers is about between $-2.207 \mathrm{E}-5 \mathrm{~K}^{-1}$ and $-2.364 \mathrm{E}-5 \mathrm{~K}^{-1}$. The experimental results are very close to the theoretical values.

\section{CONCLUSION}

The dynamic testing system for MEMS microstructures was developed, and the TCF of two single crystal silicon microcantilevers was tested. The impact base excitation method with shock wave was proved to be effective for the dynamic measurement of microstructures under the high-temperature environment. The experimental results show that under the high-temperature environment, the firstorder natural frequencies of silicon microcantilevers slightly and linearly decrease with the elevated temperature. The variation is induced by the changes in the elastic modulus and physical dimensions. Both the T-shaped microcantilever and the microcantilever with a uniform rectangular crossing section almost have the same TCF. The experimental result is very close to the theoretical analysis.

\section{ACKNOWLEDGEMENT}

This work was supported by the National Natural Science Foundation of China (Grant no. 51305191) and the General Project of Liaoning Provincial Education Department of China (grant no. L2015009).

\section{REFERENCES}

[1] Robert, B. (2004). Recent developments in MEMS sensors: A review of applications, markets and technologies. Sensor Review, vol. 33, no. 4, p. 300-304, D0l:10.1108/SR-05-2013678.

[2] Michael, K., White, N. (eds.) (2013). MEMS for Automotive and Aerospace Applications. Elsevier, Oxford.

[3] Sandberg, R., Svendsen, W., Mølhave, K., Boisen, A. (2005). Temperature and pressure dependence of resonance in multi-layer microcantilevers. Journal of Micromechanics and Microengineering, vol. 15, no. 8, p. 1454-1458, DOI:10.1088/0960-1317/15/8/011.

[4] Boyd, E.J., Li, L., Blue, R., Uttamchandani, D. (2013). Measurement of the temperature coefficient of Young's modulus of single crystal silicon and $3 \mathrm{C}$ silicon carbide below 273K using micro-cantilevers. Sensors and Actuators A: Physical, vol. 198, p. 75-80, D0l:10.1016/j.sna.2013.04.032.

[5] Chuang, W., Luger, T., Fettig, R., Ghodssi, R. (2004). Mechanical property characterization of LPCVD silicon nitride thin films at cryogenic temperatures. Journal ofMicroelectromechanical Systems, vol. 13, no. 5, p. 870-879, DOI:10.1109/JMEMS.2004.836815.
[6] Partridge A., Reynolds J.K., Chui B.W., Chow, E.M, Fitzgerald, A.M., Zhang, L., Malus, N.l., Kenny, T.W. (2000) A highperformance planar piezoresistive accelerometer. Journal of Microelectromechanical System, vol. 9, no. 1, p. 58-66, DOI:10.1109/84.825778.

[7] Xiong, X., Wu, Y.-L. D., Jone, W.-B. (2005). A dual-mode builtin self-test technique for capacitive MEMS devices. IEEE Transactions on Instrumentation and Measurement, vol. 54, no. 5, p. 1739-1750, D0I:10.1109/TIM.2005.855094.

[8] Burdess, J.S., Harris, A.J., Wood, D., Pitcher, R.J., Glennie, D. (1997). A system for the dynamic characterization of microstructures. Journal of Microelectromechanical Systems, vol. 6, no. 4, p. 322-328, D0l:10.1109/84.650129.

[9] Hart, M.R., Conant, R.A., Lau, K.Y., Muller, R.S. (2000). Stroboscopic interferometer system for dynamic MEMS characterization. Journal of Microelectromechanical Systems, vol. 9, no. 4, p. 409-418, D0l:10.1109/84.896761.

[10] Flynn, E.B., Bassman, L.C., Smith, T.P., Lalji, Z., Fullerton, L.H., Leung, T.C., Greenfield, S.R. Koskelo, A.C. (2006). Threewavelength electronic speckle pattern interferometry with the Fourier-transform method for simultaneous measurement of microstructure-scale deformations in three dimensions. Applied Optics, vol. 45, no. 14, p. 3218-3225, D0l:10.1364/ A0.45.003218.

[11] Kim, P., Bae, S., Seok, J. (2012). Resonant behaviors of a nonlinear cantilever beam with tip mass subject to an axial force and electrostatic excitation. International Journal of Mechanical Sciences, vol. 64, no. 1, p. 232-257, DOl:10.1016/j.ijmecsci.2012.06.008.

[12] Littrell, R., Grosh, K. (2012). Modeling and characterization of cantilever-based MEMS piezoelectric sensors and actuators. Journal of Microelectromechanical Systems, vol. 21, no. 2, p. 406-413, D0I:10.1109/JMEMS.2011.2174419.

[13] Wu, C.-T., Hsu, W. (2002). An electro-thermally driven microactuator with two dimensional motion. Microsystem Technologies, vol. 8, no. 1, p. 47-50, D0l:10.1007/s00542001-0129-7.

[14] Xiong, L., Zhou, Q., Wu, Y., Chen, P. (2015). New laser excitation method for modal analysis of microstructure. Mechanical Systems and Signal Processing, vol. 50-51, p. 227-234, DOl:10.1016/j.ymssp.2014.05.012.

[15] Huber, T., Hagemeyer, S., Ofstad, E., Fatemi, M., Kinnick, R., Greenleaf, J. (2007). Noncontact modal excitation of small structures using ultrasound radiation force. Proceedings of the SEM Annual Conference and Exposition on Experimental and Applied Mechanics, p. 604-610.

[16] Ricci, J., Cetinkaya, C. (2007). Air-coupled acoustic method for testing and evaluation of microscale structures. Review of Scientific Instruments, vol. 78, no. 5, p. 005105 , DOl:10.1063/1.2735574.

[17] Wang, X.D., Li, N., Wang, T., Liu, M.W., Wang, L.D. (2007). Dynamic characteristic testing for MEMS micro-devices with base excitation. Measurement Science and Technology, vol. 18, no. 6, p. 1740-1747, DOl:10.1088/0957-0233/18/6/s12.

[18] Burak Ozdoganlar, O., Hansche, B.D., Carne, T.G. (2005). Experimental modal analysis for microelectromechanicalsystems. Experimental Mechanics, vol. 45, no. 6, p. 498-506, Dol:10.1007/bf02427903. 
[19] Madou, M.J. (2011). Fundamentals of Microfabrication and Nanotechnology: Manufacturing Techniques for Microfabrication and Nanotechnology. CRC Press, Boca Raton.

[20] Pilkey, W. (1994). Formulas for Stress, Strain, and Structural Matrices. Wiley, New York.

[21] Young, W.C. (1989). Roark's Formulas for Stress and Strain. McGraw-Hill, New York.

[22] Petersen, K.E. (1982). Silicon as a mechanical material. Proceedings of the IEEE Electron Devices, vol. 70, no. 5, p. 420-457, DOI:10.1109/PROC.1982.12331.
[23] McSkimin, H.J. (1953). Measurement of elastic constants at low temperatures by means of ultrasonic waves-data for silicon and germanium single crystals, and for fused silica. Journal of Applied Physics, vol. 24, no. 8, p. 988-997, DOl:10.1063/1.1721449.

[24] Soma, T., Kagaya H.M. (1988). Properties of Silicon, EMIS Data Reviews Series, no. 4, Wiley, New York.

[25] Watanabe, H., Yamada, N., Okaji, M. (2004). Linear thermal expansion coefficient of silicon from 293 to $1000 \mathrm{~K}$. International Journal of Thermophysics, vol. 25, no. 1, p. 221236, D0I:10.1023/B:IJ0T.0000022336.83719.43. 